\title{
Caspase-7 ablation modulates UPR, reprograms TRAF2-JNK apoptosis and protects T17M rhodopsin mice from severe retinal degeneration
}

\author{
S Choudhury ${ }^{1}$, Y Bhootada ${ }^{1}, 0$ Gorbatyuk ${ }^{2}$ and M Gorbatyuk ${ }^{*, 1}$
}

The UPR is activated in the mouse retina expressing misfolded T17M rhodopsin (RHO) during autosomal dominant retinitis pigmentosa (ADRP) progression. Therefore, the goal of this study is to validate the UPR-induced caspase-7 as a new therapeutic target that modulates the UPR, reduces the level of apoptosis and protects the ADRP retina from retinal degeneration and light-induced damage. Mice were analyzed using ERG, SD-OCT and histology to determine the role of caspase-7 ablation. The results of these experiments demonstrate the significant preservation of photoreceptors and their function in T17M RHO CASP-7 retinas from P30 to P90 compared with control mice. These mice were also protected from the light-induced decline in the ERG responses and apoptosis. The RNA and protein analyses of T17M RHO + Csp7-siRNA, Tn + Csp7-siRNA 661W cells and T17M RHO CASP-7 retinas revealed that caspase-7 ablation reprograms the UPR and reduces JNK-induced apoptosis. This reduction is believed to occur through the downregulation of the mTOR and Hif1a proteins. In addition, decline in activated PARP1 was detected in T17M RHO CASP-7 retina. Altogether, our findings indicate that the targeting of caspase-7 in T17M RHO mice could be a feasible therapeutic strategy for advanced stages of ADRP.

Cell Death and Disease (2013) 4, e528; doi:10.1038/cddis.2013.34; published online 7 March 2013

Subject Category: Experimental medicine

The T17M mutation within the Rhodopsin $(R H O)$ gene, which substitutes a Thr with a Met at position 17, affects the assembly of the opsin protein with 11 -cis-retinal ${ }^{1}$ and presumably impairs protein stability, folding and trafficking, ${ }^{1,2}$ leading to a severe form of retinal degeneration known as autosomal dominant retinitis pigmentosa (ADRP). It has been proposed that ADRP photoreceptors, in general, ${ }^{3}$ and $\mathrm{T} 17 \mathrm{M}$ $R H O$, in particular, ${ }^{4}$ die through apoptosis. Recently, we have shown that endoplasmic reticulum (ER) stress is involved in the mechanism of S334ter, $\mathrm{P} 23 \mathrm{H}$ and T17M RHO photoreceptor death..$^{5-7}$ However, it has not yet been proven that triggering the UPR causes ADRP photoreceptor death. The contribution of the ER stress-induced caspase-7 to apoptosis has been controversial until very recently. ${ }^{8-10}$ Because the structure of caspase $-7^{9}$ exhibits a high degree of similarity with caspase- $3,{ }^{11}$ it was believed that the role of caspase- 7 is redundant with that of caspase-3, thus minimizing the impact of caspase-7 on the apoptotic cascade. However, it was later determined that owing to the presence of a unique negative electrostatic potential in the $\mathrm{S} 4$ region of the catalytic site of caspase-7, it has different substrates than caspase-3. ${ }^{11}$ There are at least four known caspase- 7 targets that are not shared by caspase-3: caspase-12, kinectin, TNFRI and $\mathrm{p} 23 .{ }^{11,12}$

Despite the fact that caspase- 7 knockout mice have a normal appearance, organ morphology and lymphoid development, ${ }^{13}$ recent studies strongly suggest that caspase- 7 has an important, non-redundant role in normal physiology and apoptotic cell death. For example, Le et al. ${ }^{14}$ found no evidence of any compensatory activation of caspase-7 in the CNS following in vivo cerebral ischemia in CASP-3-deficient mice. In addition, the treatment of human neuroblastoma SH-SY5Y cells exposed to the anticancer apoptotic-inducing drug paclitaxel, the inhibitor of activated caspase-7, results in a modulation of the apoptotic signals, suggesting that caspase-7 and caspase-3 have complementary but not completely overlapping roles. ${ }^{15}$ The possible role of caspase-7 in the regulation of hypoxia-induced apoptosis as well as the relationship between caspase-7 and the PARP cleavage that is known to occur in ADRP retinas ${ }^{16}$ have been

\footnotetext{
${ }^{1}$ Department of Cell Biology and Anatomy, North Texas eye Research Institute, University of North Texas Health Science Center, 3500 Camp Bowie Blvd, Fort-Worth, TX 76107, USA and ${ }^{2}$ Department of Molecular Genetics and Microbiology, University of Florida, 2033 Mowry Road, Gainesville, FL 32610, USA

${ }^{*}$ Corresponding author: M Gorbatyuk, Department of Cell Biology and Anatomy, North Texas eye Research Institute, University of North Texas Health Science Center, 3500 Camp Bowie Blvd, Fort-Worth, TX 76107, USA. Tel: + 817735 2682; Fax: + 817735 2637; E-mail: mgortk@uab.edu

Keywords: retinal degeneration; hT17M RHO; UPR; caspase-7; apoptosis

Abbreviation: RHO, Rhodopsin; ER, endoplasmic reticulum; CNS, central nervous system; PARP, Poly (ADP-ribose) polymerase; UPR, unfolded protein response; ERG, electroretinogram; SD-OCT, spectral domain optical coherence tomography; ONL, outer nuclear layer; T17M RHO CASP-7, T17M Rhodopsin Caspase-7 - / - ; siRNA, small interfering RNA; mRNA, messenger RNA; Atf4, activating transcription factor 4; Atf6, activating transcription factor 6; Bip, binding immunoglobulin protein; CHOP, CCAAT/-enhancer-binding protein homologous protein; Bax, Bcl-2-associated X protein; Hif1a, hypoxia-inducible factor 1; mTor, mammalian target of rapamycin; Traf2, TNF receptor-associated factor 2; PARP1, Poly (ADP-ribose) polymerase 1; pATF6, phosphorylated activating transcription factor 6; Casp12, Caspase-12; IRE1, inositol-requiring enzyme 1; Edem2, ER degradation enhancer, mannosidase alpha-like 2; Hsp90, heat shock protein 90; CNX, Calnexin Bim- bcl-2interacting mediator of cell death; Bik, Bcl2-interacting killer; Akt, protein kinase B (PKB); TNF, tumor necrosis factor; RT-PCR, reverse transcriptase-polymerase chain reaction; ELISA, enzyme-linked immunosorbent assay; SDS-PAGE, sodium dodecyl sulfate-polyacrylamide gel electrophoresis

Received 02.7.12; revised 01.1.13; accepted 18.1.13; Edited by A Verkhratsky
} 
recently investigated. ${ }^{17}$ All of the above-mentioned studies point out the therapeutic outcome that could be achieved from the ablation of caspase-7.

Current pharmacotherapies for ADRP include dietary supplementation with vitamin $A$ and docosahexaenoic acid. However, gene therapy, with its ability to turn off or replace mutated genes has been developed as an attractive alternative approach. ${ }^{6,18}$ In addition, an indirect approach for promoting photoreceptor cell survival and targeting apoptosis without affecting the expression of the mutant protein, especially at late stages of the ADRP progression, should be taken in consideration as well. ${ }^{6}$ This is particularly important for those ADRP photoreceptors that are close to passing the point of no return along the self-destruction pathway. The 'suppression and replacement' strategy ${ }^{19}$ alone may not be a viable approach for these cells, and only the combination of two approaches for modulating the activated UPR at the level of the misfolded $\mathrm{RHO}$ and the UPR-induced apoptosis will be beneficial in treating ADRP. Therefore, targeting caspase-7 might be a promising therapy for maintaining ADRP photoreceptor function and integrity.

Thus, the goal of the current study was to (1) verify whether the modulation of the targets downstream of the activated UPR is a feasible therapeutic approach for ADRP treatment leading to a lower level of apoptosis; (2) validate the caspase7 gene as a new therapeutic target for ADRP photoreceptor survival; and (3) elucidate the molecular mechanisms underlying the link between caspase-7 ablation and the cellular signaling involved in the preservation of vision in T17M RHO retinas. If it is successful, the proposed approach aimed at reducing apoptosis could be used to treat advanced stages of ADRP either alone or in combination with a 'suppression and replacement' strategy reducing the level of misfolded $\mathrm{RHO}$. This approach may also be applicable to the treatment of other ocular diseases.

\section{Results}

The expression and activation of caspase-7 in $\mathrm{T} 17 \mathrm{M}$ $\boldsymbol{R H O}$ retina. Our previous study found that caspase-7 is activated during the progression of ADRP. ${ }^{6}$ Therefore, we examined the RNA extract of T17M RHO retina and found that caspase-7 gene expression was dramatically increased by 2.7-fold beginning at P18 (Figure 1a; Supplementary Figure S1 and Supplementary Table S1). At P21 and P25, the caspase-7 gene expression was upregulated in the T17M $R H O$ retina 3.2 -fold and 3.95 -fold, respectively. This upregulation resulted in a 4.5-fold increase in the activation of the caspase-7 protein at P21 (Figure 1b; Supplementary Figure S1 and Supplementary Table S1) leading to a 3.6-fold elevation in a ratio of cleaved-to-uncleaved caspase-7.

The functional rescue of photoreceptors in T17M RHO mice by caspase-7 ablation. To test the function of $T 17 \mathrm{M}$ $\mathrm{RHO}$ photoreceptors, we registered the a- and b-waves of the scotopic ERG response at P30, P60 and P90. Figure 2a, Supplementary Figure S3 and Supplementary Table S1 show the results of this analysis, which suggest that during these 3 months, the a-wave amplitude in T17M RHO CASP-7 was increased from 166-478\% compared with T17M RHO at P30 and P90, respectively. The b-wave of the scotopic ERG amplitude was also dramatically elevated in T17M $R H O$ CASP-7 to $145 \%$ and $182 \%$ at $\mathrm{P} 30$ and P90, respectively. However, this rescue was partial: the a- and b-wave amplitudes in P30, 60 and 90 T17M RHO CASP-7 were 41\%, 48\%, $41 \%$ and $67 \%, 73 \%, 59 \%$ respectively, compared with wt.

The preservation of retinal structural in T17M RHO mice by caspase-7 ablation. The SD-OCT analysis revealed (Figure 3; Supplementary Figure S4 and Supplementary Table S1) that the thickness of the outer nuclear layer (ONL) in the inferior retina in T17M RHO CASP-7 mice was increased compared with $\mathrm{T} 17 \mathrm{M}$ RHO to $168 \%$ and $298 \%$ at P30 and P90, respectively. The thickness of the ONL in the superior retina was also significantly increased compared with $\mathrm{T} 17 \mathrm{M}$ RHO from $166 \%$ at P30, to $268 \%$ at P30 and P90, respectively. Despite the significant increase of the ONL thickness, this rescue was partial and was $82 \%, 73 \%, 61 \%$ and $80 \%, 76 \%, 59 \%$ of the ONL thicknesses in wt superior and inferior retina at $\mathrm{P} 30, \mathrm{P} 60$ and P90, respectively.
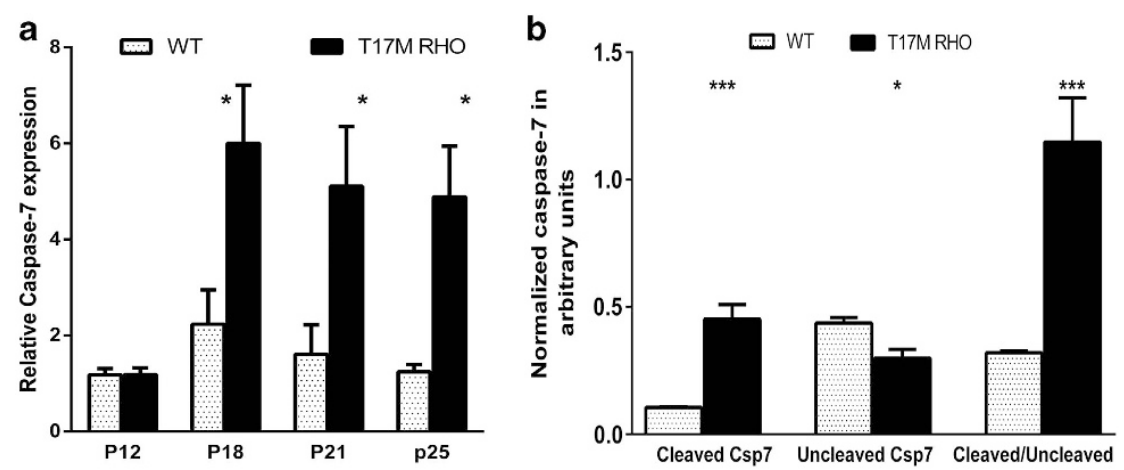

Figure 1 The expression and activation of caspase-7 (Csp7) in the T17M RHO retina. Four animals were used for each group in this experiment. (a) Starting at P18, we observed a 2.7-fold $(P<0.05)$ increase in the Csp7 mRNA in T17M RHO mice compared with wt. At P21 and P25, Csp7 gene expression was upregulated to between 3- and 4-fold. (b) At P21, we detected a 4.5-fold $(P<0.0008)$ increase in the activation of the Csp7 protein in T17M RHO retinas (Supplementary Figure S1 and Supplementary Table S1) leading to a 3.6-fold elevation in the ratio of cleaved-to-uncleaved Csp7 and suggesting that the ADRP photoreceptors experience the activation of Csp7. However, because the photoreceptors are not the only cells that contribute to the retinal protein extract, the possibility exists that other retinal cell types such as bipolar, RGC and Muller cells may be involved in the activation of Csp7. Therefore, the precise retinal cell types that are inducing and activating Csp7 will need to be addressed in the future. Data are shown as mean \pm S.E.M. (Supplementary Table S1) $\left({ }^{*} P<0.05,{ }^{* * *} P<0.001\right)$ 
a

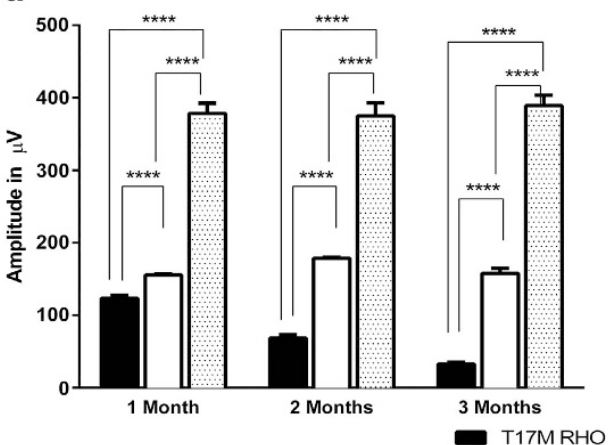

b

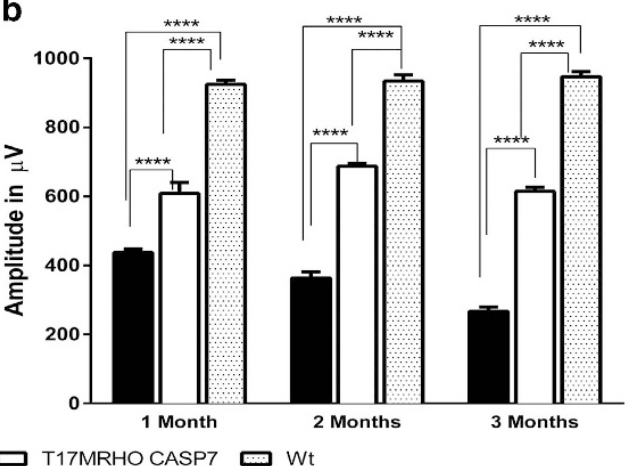

Figure 2 The lack of caspase-7 protects T17M RHO retinas from reduced scotopic ERG responses. Six animals were used for each group in this experiment. Different groups of animals were used in the longitudinal study. a- and b-wave amplitudes in the scotopic ERG of 1-, 2- and 3-month-old mice were calculated. The $P$-values between all groups were statistically significant $(P<0.0001)$. Data are shown as mean \pm S.E.M. and are also presented in Supplementary Table S1. (a) The T17M RHO mice demonstrated a steady decline in a-wave amplitudes from 94 to $33 \mu \mathrm{V}$ during 3 months, suggesting that they were experiencing severe retinal degeneration. Alternatively, the T17M RHO CASP-7 retinas showed a preservation of retinal function. The a-wave amplitude was increased by $166 \%$ in T17M RHO CASP-7 compared with T17M RHO at $\mathrm{P} 30$, by $261 \%$ at P60, and by $478 \%$ at P90. (b) The b-wave amplitudes continuously declined in T17M RHO mice from 432 to $307 \mu \mathrm{V}$, while the T17M RHO CASP-7 retinas increased by $145 \%$ at P30, by $177 \%$ at P60 and by $182 \%$ at P90. Analysis of the a- and b-wave amplitudes suggests that caspase- 7 ablation essentially blocks the retinal degeneration in T17M RHO retinas $\left({ }^{* * *} P<0.0001\right)$

The OCT data were confirmed by histology (Supplementary Figure S2), which demonstrated reduction in the ONL nuclei in the 3-month-old T17M RHO retina compared with 1-monthold (5.4 \pm 0.32 rows versus $7.9 \pm 0.16)$. During this period, the T17M RHO CASP-7 animals did not show the same degree of progressive photoreceptor death, although there was an $18 \%$ decline in the numbers of photoreceptors as compared with wt (Supplementary Figure S2). There was no notable difference in the $\mathrm{RHO}$ immunoreactivity or organization of the inner and outer segments in these groups.

The T17M $R H O$ retina lacking caspase-7 is less sensitive to light-induced damage. It has been shown that the T17M RHO mice are sensitive to light. ${ }^{4}$ Therefore, we decided to investigate whether the caspase- 7 ablation protects these retinas from light-induced damage. Analysis of a-wave amplitudes of the experimental (right eye) to control eye (left eye) indicated a $33 \%$ reduction in T17M $R H O$ retina compared with wt measures at $15 \mathrm{~dB}$ (Figure 4a; Supplementary Table S1). The caspase-7 ablation in these mice preserved the function of ADRP photoreceptors and rescued the loss of a-wave amplitude by $43 \%$ as compared with $\mathrm{T} 17 \mathrm{M} \mathrm{RHO}$ retinas.

To evaluate the cellular stress induced by light exposure, we also performed a nucleosome release assay in which we detected the apoptotic signal measured by DNA fragmentation (Figure 4b; Supplementary Table S1). We found that in the right eyes of $\mathrm{T} 17 \mathrm{M} \mathrm{RHO}$ mice, light exposure leads to a 3.8-fold increase in the apoptotic signal compared with wt. The T17M RHO CASP-7 retina, however, demonstrated a significant reduction in the apoptotic signal by $65 \%$ compared with $\mathrm{T} 17 \mathrm{M} R H O$. The difference between the apoptotic signals measured in wt and T17M RHO CASP-7 was not significant.

The knock-down of caspase-7 in $661 \mathrm{~W}$ cells expressing T17M RHO leads to a reprogramming of the UPR-associated gene expression and JNK-activated apoptosis. To study the mechanism by which caspase-7 ablation in T17M RHO photoreceptors leads to a therapeutic effect, we transfected the retinoblastoma cone-derived $661 \mathrm{~W}$ cells with a plasmid expressing the human wtRHO (pCMV6$A C$-wtRHO-GFP) and T17M RHO protein fused with GFP (pCMV6-AC-T17M RHO-GFP) and either siRNAs targeting caspase-7 (Csp7-siRNA) or control siRNA. The results of this analysis are shown in Figure 5a; Supplementary Table S1.

Our recent study using T17M RHO mice demonstrated that the activated UPR is involved in retinal degeneration in these animals. ${ }^{7}$ Therefore, we decided to test whether the therapeutic effect triggered by caspase- 7 ablation in transgenic retinas is associated with the modulation of the UPR. To verify this link, in vitro we analyzed the UPR-associated gene expression and found that in T17M RHO + Csp7-siRNA with $92 \%$ knockdown of caspase-7 mRNA (0.05 \pm 0.002 versus $0.61 \pm 0.014$ in contr. siRNA, $\left.P=7.72 \times 10^{-9}\right)$, the UPR-induced gene expression was modulated compared with control cells (T17M RHO + cnt.siRNA) and was not significantly different compared with wtRHO. For instance, the relative gene expression of Atf4, Atf6, Bip and CHOP were reduced by 55\%, 50\%, 61\% and 31\% in T17M RHO + Csp7siRNA cells compared with T17M RHO + cnt.siRNA cells, respectively. Expression of other UPR-associated genes, such as Bax, Hif1a, mTor, Traf2 and c-Jun, were also downregulated in experimental cells by $49 \%, 53 \%, 46 \%$, $53 \%$ and $43 \%$, respectively.

We also verified the modulation of the activated UPR markers by western blots (Figure 5b; Supplementary Figure S1 and Supplementary Table S1) and found that the level of the UPR-associated proteins in T17M RHO + Csp7-siRNA cells was modified compared with control and was not different compared with wtRHO + cnt.siRNA. For example, we found that the level of cleaved pAtf6 protein $(50 \mathrm{kDa})$, Bip, cleaved Csp12, mTOR was significantly reduced by $40 \%, 58 \%, 31 \%$ and $30 \%$, respectively.

Because of our preliminary data showing the activation of light-induced apoptosis and previously reported activation of the IRE pathway in T17M RHO retinas, ${ }^{7}$ we choose to 
a

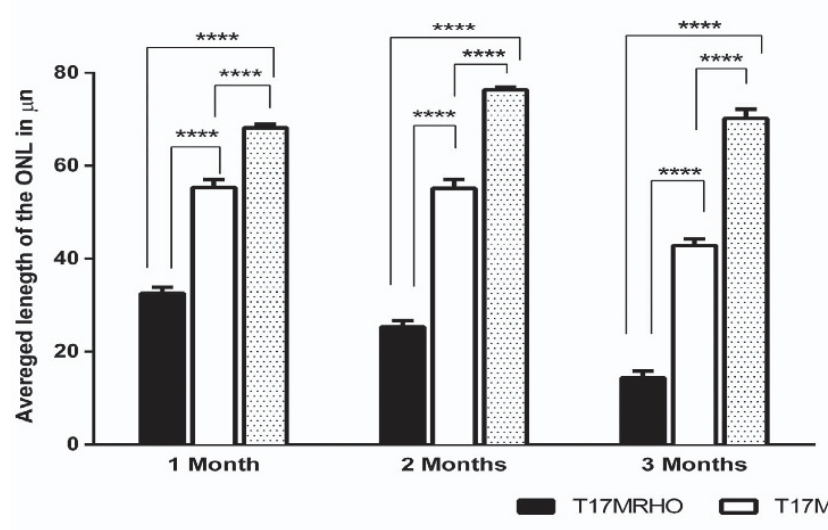

c

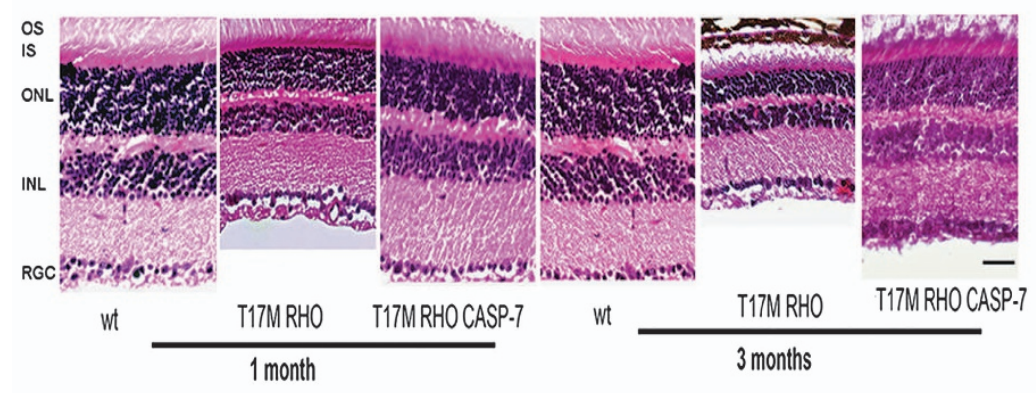

b

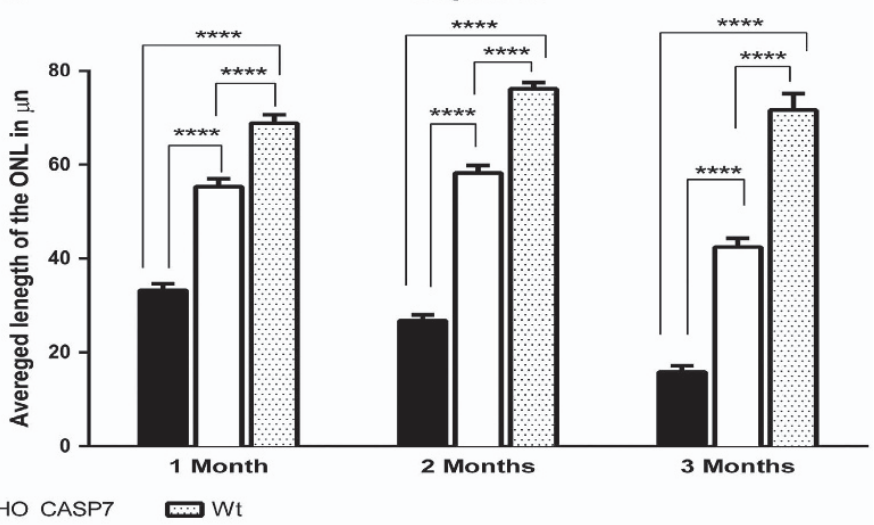

Figure 3 The preservation of retinal structure in T17M RHO CASP-7 retina measured by SD-OCT analysis. Significant changes in the average thickness of the ONL in the inferior and superior hemispheres in 1-, 2- and 3-month-old mice were found. The $P$-values between all groups were calculated using a two-way ANOVA and were statistically significant $(P<0.0001)$. Data from six animals in each group are shown as mean \pm S.E.M are resented in Supplementary Table S1.(a) The SD-OCT analysis demonstrated a significant decline in the average thickness of the ONL in T17M RHO inferior retinas, and their thickness was reduced by $24 \%$ at 2 months compared with 1 month and by $58 \%$ at 3 months compared with 1 month of age, which is $80 \%$ lower than that in wt. In the T17M RHO CASP-7 mice, however, we observed a significant increase in the average thickness of the inferior ONL by $168 \%$ at P30, by $221 \%$ at P60 and by $298 \%$ at P90, suggesting the preservation of retinal structure in T17M RHO CASP-7 inferior retinas. (b) The SD-OCT analysis showed a significant decline in the average thickness of the superior T17M RHO retinas that was 20\% lower at 2 months compared with 1 month and $53 \%$ lower at 3 months compared with 1 month of age, which is $78 \%$ lower than in wt. In the T17M RHO CASP-7 mice, however, we observed a significant increase in the average thickness of the ONL in the superior hemispheres by $166 \%$ at P30, by $217 \%$ at P60 and by $267 \%$ at P90. In a separate study, analyzing the difference between 1-, 2and 3-month timepoints in the inferior and superior T17M RHO CASP-7 retina (by one-way ANOVA), we found that all regions exhibited a difference in the thickness of the ONL between 2 and 3 months as well as 1- and 3-month-old retinas. However, from our histological analysis with hematoxylin and eosin (H\&E) staining (see below), we showed that there was no significant decline in the number of rows of T17M RHO CASP-7 photoreceptors between 1 and 3 months. The discrepancy in trends between the two different methods of evaluation of retinal structure suggests that long-lasting morphological changes occur before the photoreceptor cell death. (c) Histological analyses of wt, T17M RHO and T17M RHO CASP-7 retinas: images of wt, T17M RHO, T17M RHO CASP-7 retinas stained with H\&E. Five animals in each group were used in this experiment. Histology of experimental mouse retinas at 1 and 3 months of age showed loss of photoreceptor cell nuclei, a shortening of the outer segments and general disorganization in the T17M RHO retina that was not that pronounced in the T17M RHO CASP-7 mice. GCL, ganglion cell layer; INL, inner nuclear layer; ONL, outer nuclear layer; IS, inner segments; OS, outer segments. Scale bar indicates $30 \mu \mathrm{m}$. (d) Photoreceptor cell nuclei in all groups of animals. The number of nuclei was counted by a masked researcher. Two-way ANOVA with multiple comparison analysis demonstrated differences in allthree 3 groups of animals $\left.{ }^{* * * *}, P<0.0001\right)$ at 1 and 3 months of age with the exception of comparison between wt mice of 1 and 3 months and T17M RHO CASP-7 mice of 1 and 3 months. For example, 1-month-old T17M RHO mice had $7.9 \pm 0.16$ rows and this number was significantly reduced by 3 months of age to $5.38 \pm 0.32$ rows. The T17M RHO CASP-7 mice had less sever loss of photoreceptor cells: $9.4 \pm 0.16$ at 1 month and $9.23 \pm 0.12$ at 3 months. However, these numbers were significantly different from the wt animals $(11.39 \pm 0.16$ at 1 month and $11.45 \pm 0.12$ at 3 months). The histological analysis confirmed our OCT data suggesting first, that there is a decline in the number of photoreceptor cells in T17M RHO at 1 and 3 month by $68 \%$ and second, the caspase-7 ablation delays degeneration of the T17M RHO retina. Decline in the number of photoreceptors in these animals was $18 \%$ compared with wt. The rescue in T17M RHO CASP-7 was partial. For example, despite the significant increase of the ONL thickness this rescue of T17M RHO CASP-7 retinas was $82 \%$, $73 \%$, $61 \%$ (inferior) and $80 \%, 76 \%, 59 \%$ (superior) of the ONL thicknesses in wt at P30, P60 and P90, respectively. In favor of this hypothesis, by a separate one-way ANOVA comparison, we also found that there is no difference between ERG amplitudes in 1- and 3-month-old retinas suggesting that these photoreceptors are functioning at similar levels $\left.{ }^{* * * *} P<0.0001\right)$

analyze the p-c-Jun protein, which is known to be activated through a recruitment of the TRAFf2 protein by IRE1 Figure 5b; Supplementary Figure $\mathrm{S} 1$ and Supplementary Table 1S). We found that the level of $p$-c-Jun protein was significantly increased by $57 \%$ in T17M RHO + cnt.siRNA cells compared with wtRHO + cnt.siRNA cells and was significantly diminished by $43 \%$ in T17M RHO + Csp7-siRNA cells compared with $\mathrm{T} 17 \mathrm{M} \mathrm{RHO}$ control.

Wondering whether or not the effect of caspase-7 ablation in cells experiencing the activation of the UPR is specific to 
a

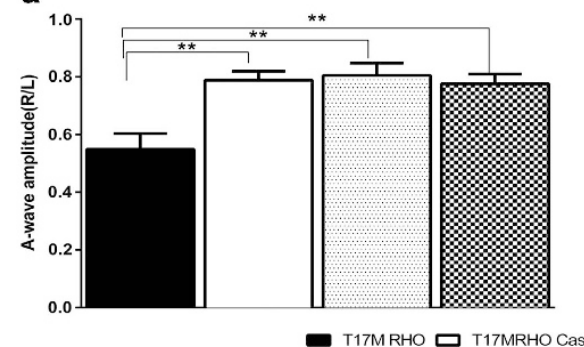

b

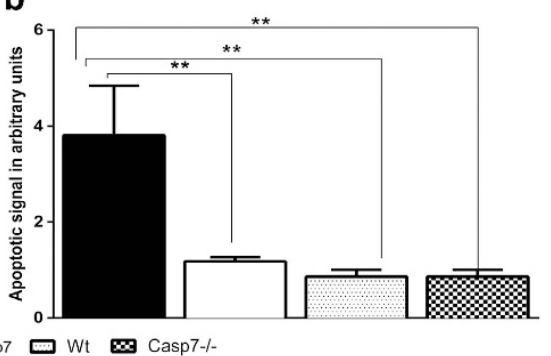

Figure 4 The scotopic ERG amplitudes are protected and the activation of apoptosis is inhibited in light-exposed P30 T17M RHO CASP-7 retinas. Data from seven animals in each group are shown as mean \pm S.E.M. in Supplementary Table S1. (a) The R/L ratio of the a-waves was used to evaluate the difference between groups. It was statistically significant for all comparisons calculated using a one-way ANOVA with the exception of wt versus T17M RHO CASP-7; T17M RHO CASP-7 versus CASP-7 and CASP- 7 versus wt retinas. These results suggest that the caspase-7 ablation protects the T17M RHO retinas from accelerated vision loss triggered by light exposure resulting in an increase in the R/L a-wave amplitude by $43 \%$. (b) The R/L ratio was used to analyze the apoptotic signal triggered by light exposure using a one-way ANOVA. The animals were killed after performing ERG analysis and retinal extracts were used to analyze the apoptotic signal in right and left retinal extract. The apoptotic signal in T17M RHO retinas was 3.8-fold higher than in wt. The T17M RHO CASP-7 animals demonstrated a 65\% lower apoptotic signal compared with the T17M RHO mice and no significant difference compared with wt. No difference was also detected when comparing the CASP-7 versus wt retinas. The results of this experiment demonstrated that caspase-7 is involved in light-induced damage of the T17M RHO retina and its ablation protects retina from accelerated apoptotic signal induced by light exposure $\left.{ }^{* \star} P<0.01\right)$
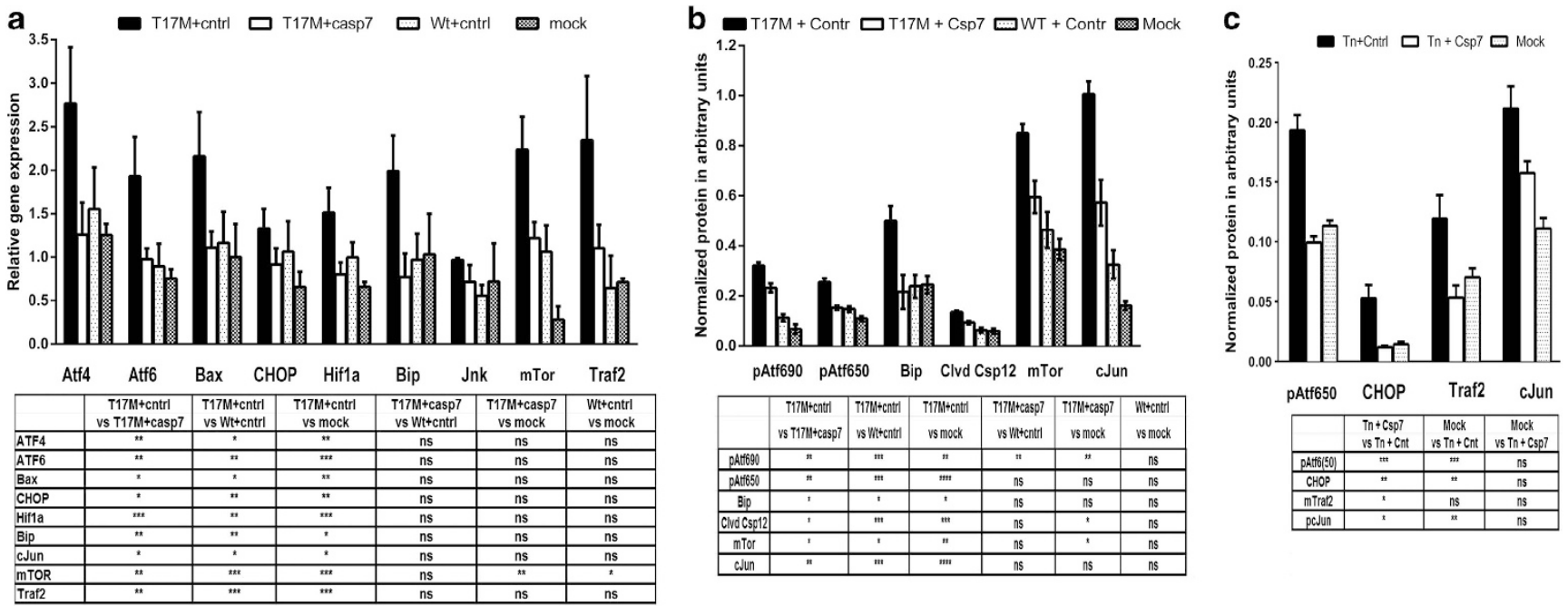

Figure 5 The knockdown of caspase-7 leads to the modulation of UPR-associated gene and protein expression in 661W cells experiencing activation of the UPR as a result of expression of misfolded RHO or treatment with tunicamycin. Results of this experiment are also presented in Supplementary Table S1. (a) RT-PCR analysis of cells expressing the T17M RHO and co-tranfected with cont.siRNA or siRNA against caspase-7. In T17M RHO Csp7-siRNA cells we observed that the UPR-induced gene expression was modulated compared with control cells (T17M RHO + cnt.siRNA). For instance, the relative gene expression of Att4, Atf6, Bip and CHOP were reduced by 55, 50, 61 and $31 \%$ compare to T17M RHO + cnt.siRNA cells, respectively. Expression of other UPR-associated genes, such as Bax, Hif1a, mTor, Traf2 and c-Jun, was also downregulated in experimental cells by $49 \%, 53 \%, .46 \%, 53 \%$ and $43 \%$, respectively. The modulation in the UPR-induced gene expression in T17M RHO Csp7-siRNA cells was not significantly different compared with wtRHO + cnt.siRNA cells. Four different replicates of a transfection in each control and experimental groups were used to perform the experiment. (b) Western blot analysis of cells expressing the T17M RHO and cotranfected with cont.siRNA or siRNA against caspase-7. We found that that the level of the UPR-associated proteins in T17M RHO + Csp-7siRNA cells was significantly modified compared with T17M RHO + cnt.siRNA and was not different compared with wtRHO + cnt.siRNA cells. A cleaved pAtf6 protein $(50 \mathrm{kDa})$, Bip, cleaved caspase-12, mTOR, pc-Jun were significantly reduced by $40 \%, 58 \%, 31 \%, 30 \%$ and $43 \%$, respectively. Four different replicates of a transfection in each control and experimental groups were used to perform the experiment. (c) The $661 \mathrm{~W}$ cells initially transfected with Csp7-siRNA were treated with tunicamycin, the UPR inducer. Four different replicates of the transfection in each control and experimental groups were used to perform the experiment. Western blot analysis demonstrated that the caspase-7 ablation leads to downregulation of the UPR-associated cleaved pATF6 (50) and CHOP proteins by $49 \%$ and $79 \%$, respectively and reduction of $\mathrm{mTraf2}$ and pc- Jun proteins by $56 \%$ and $26 \%$, respectively. These results suggest a much more general role for caspase- 7 than modulation of cellular signaling in ADRP photoreceptors and much broader potential applications in UPR regulation. Four replicates were used for control and experimental groups $\left({ }^{\star} P<0.05,{ }^{* \star} P<0.01,{ }^{* \star *} P<0.001\right)$

T17M RHO, we performed an experiment with $661 \mathrm{~W}$ cells initially transfected with cnt. or Csp7-siRNA and subsequently treated with tunicamycin (Figure 5c; Supplementary Figure S1 and Supplementary Table S1). The results demonstrated that knocking down of caspase-7 significantly reduced the levels of
pAtf6-50, CHOP, mTraf2 and pc-Jun proteins by $49 \%, 79 \%$, $56 \%$ and $26 \%$, respectively.

Caspase-7 ablation in T17M RHO retina modulates UPR signaling. The next question we asked was whether 

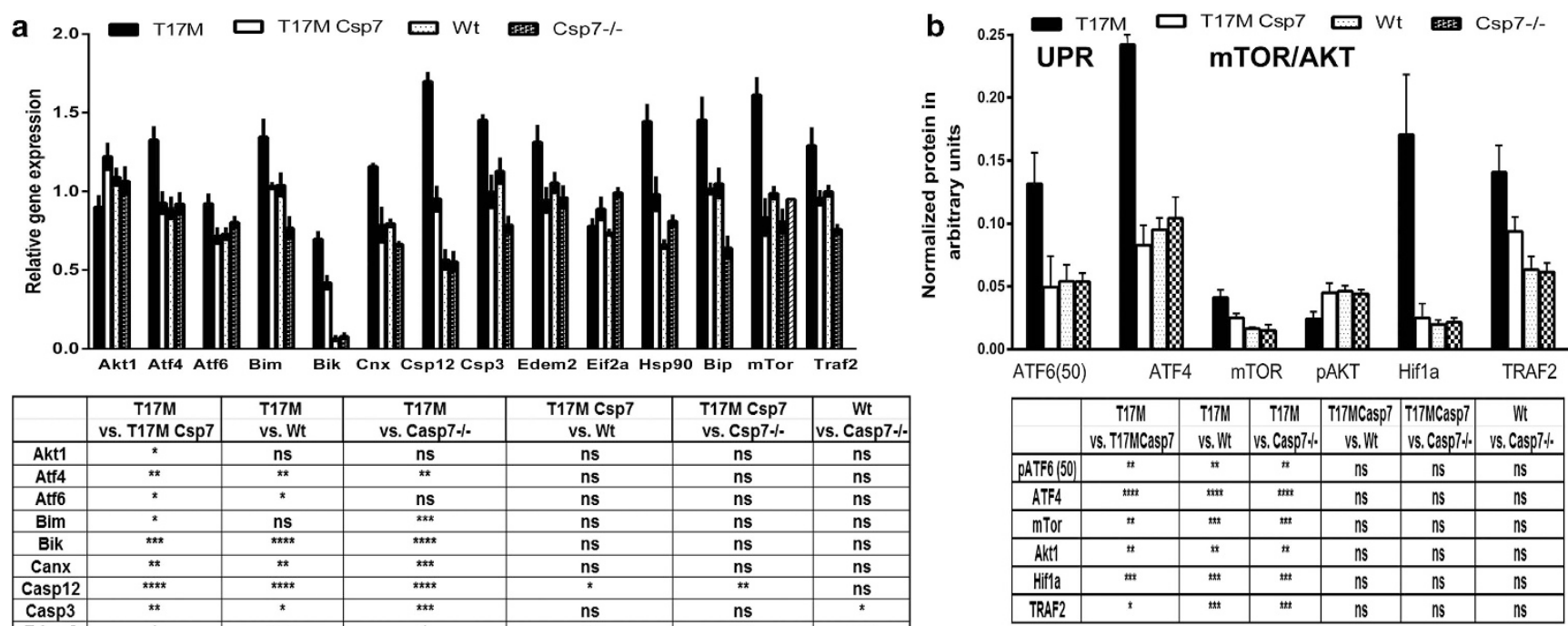

\begin{tabular}{|c|c|c|c|c|c|c|}
\hline & $\begin{array}{c}\text { T17M } \\
\text { vs. T17M Csp7 }\end{array}$ & $\begin{array}{l}\text { T17M } \\
\text { vs. Wt }\end{array}$ & $\begin{array}{c}\text { T17M } \\
\text { vs. Casp7\% }\end{array}$ & $\begin{array}{c}\text { T17M Csp7 } \\
\text { vs. Wt }\end{array}$ & $\begin{array}{l}\text { T17M Csp7 } \\
\text { vs. Csp7\% }\end{array}$ & \begin{tabular}{|c|} 
Wt \\
vs. Casp7-..
\end{tabular} \\
\hline Akt1 & * & ns & ns & ns & ns & ns \\
\hline Atf4 & $\star *$ & ** & $\star \star *$ & ns & ns & ns \\
\hline Atf6 & * & * & ns & ns & ns & ns \\
\hline Bim & * & ns & 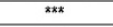 & ns & ns & ns \\
\hline Bik & $* \star *$ & $* * \pi *$ & $* * * *$ & ns & ns & ns \\
\hline Can $x$ & ** & ** & *** & ns & ns & ns \\
\hline Casp12 & ***** & 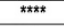 & $\star * \star \star \star *$ & * & ** & ns \\
\hline Casp3 & ** & * & $* * *$ & ns & ns & * \\
\hline Edem2 & * & ns & * & ns & ns & ns \\
\hline Eif2a & ns & ns & * & ns & ns & ns \\
\hline Hsp90 & ** & 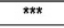 & ** & ns & ns & ns \\
\hline Bip & * & * & $\star \star * *$ & ns & ns & ns \\
\hline mTOR & $\star \star \star x$ & * & *** & ns & ns & ns \\
\hline Traf2 & * & * & $* \star *$ & ns & ns & ns \\
\hline
\end{tabular}

Figure 6 Caspase-7 ablation in T17M RHO retina reprograms the UPR signaling and modulated UPR-associated gene expression. Four animals in each group were tested. Results of the experiment are also presented in Supplementary Table S1. (a) Caspase-7 ablation leads to modulation of UPR-associated gene expression. The RNA extracts from P30 wt, T17M RHO, T17M RHO CASP-7 and CASP-7 retinas were tested. We found that the Bip, Atf4, Atf6, Cnx, Bik, Bim, Edem2, and Hsp90 $\alpha$ were downregulated in the T17M RHO CASP-7 retina by $30 \%, 30 \%, 23 \%, 33 \%, 41 \%, 23 \%, 28 \%$ and $31 \%$, respectively, compared with T17M RHO retina. The Akt1 mRNA was upregulated by $35 \%$ and the mTor mRNA was downregulated by $49 \%$. The TRAF2a mRNA expression was reduced by $27 \%$. The apoptotic caspase- 12 and caspase-3 mRNAs were downregulated by $44 \%$ and $32 \%$, respectively. (b) Western blot analysis of the retinal protein extract. The protein extracts from P30 wt, T17M RHO, T17M RHO CASP-7 and CASP-7 retinas were tested. We found that the UPR-induced proteins such as cleaved pATF6 (50) and ATF4 were elevated in T17M RHO retinas by $242 \%$ and $243 \%$, respectively. In T17M RHO CASP-7, their levels were decreased by $57 \%$ and $55 \%$, respectively. In these retinas, the expression of the pro-survival gene pAkt was increased by $60 \%$, whereas in contrast, mTOR protein expression was downregulated by $38 \%$. The TRAF2 protein level was also elevated by $217 \%$ in T17M RHO retinas. Ablation of caspase- 7 led to significant downregulation by $31 \%$ of this protein. The Hif1a protein was slightly elevated in T17M RHO retinas. However, in T17M RHO CASP-7 retina, we observed dramatic downregulation of Hif1a by $84 \%$ that was significantly lower compared even with wt retina ( ${ }^{\star} P<0.05,{ }^{* \star} P<0.01,{ }^{* \star \star} P<0.001,{ }^{* \star \star \star} P<0.0001$ )
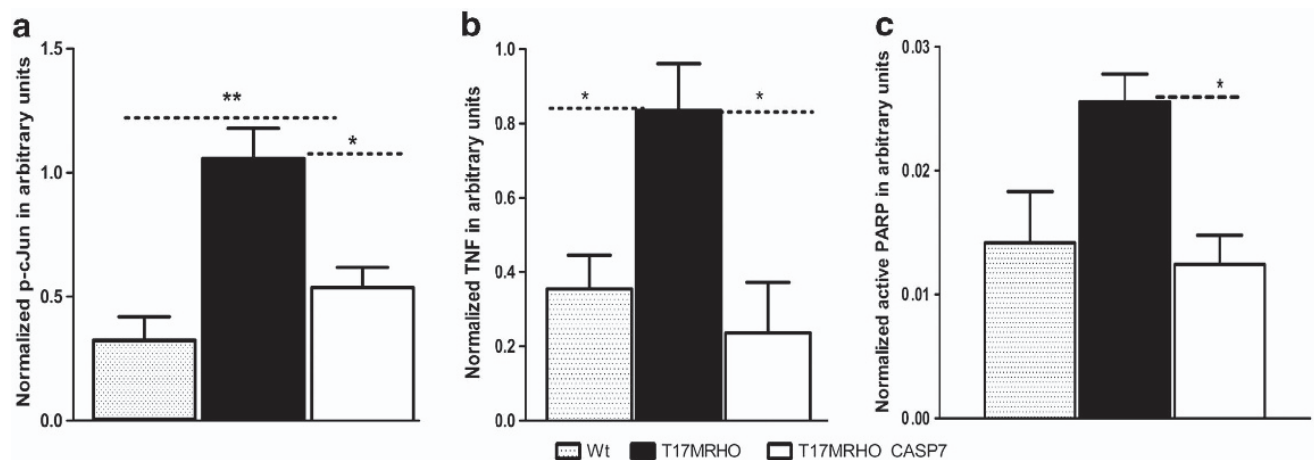

d

Figure 7 Cell death markers detected in ADRP retina by western blot analysis. Four animals were tested in each group. (a) Modulation of apoptotic marker pc-JUN. A significant increase in the pc- Jun protein was found between wt and T17M RHO $(236 \%)$, whereas caspase-7 ablation led to its reduction ( $50 \%)$, which was not statistically different compared with wt. (b) Caspase-7 ablation in the T17M RHO retinas induced reduction in the level of TNF. We found that the level of TNF in T17M RHO retinas was dramatically increased by $235 \%$ when compared with wt. The lack of caspase- 7 led to a $72 \%$ reduction in ADRP retinas and was not significantly different compared with wt. (c) Activated PARP1 was found to be elevated 1.8-fold in ADRP retinas. Caspase-7 ablation led to a reduction of activated PARP1 in T17M RHO mice by 52\% that was not statistically different from wt retinas. (d) Representative images of western blot treated with pc-JUN, TNF and PARP $\left({ }^{*} P<0.05,{ }^{* *} P<0.01\right)$

caspase-7 ablation is able to modulate the UPR-induced gene expression in T17M RHO retina. Figure 6 illustrates that the mRNA expression of the Bip, Atf4, Atf6, Cnx, Bik, Bim, Edem2 and Hsp90 $\alpha$ were downregulated in the T17M RHO CASP-7 retina by $30 \%, 30 \%, 23 \%, 33 \%, 41 \%, 23 \%, 28 \%$ and $31 \%$, respectively. The Akt1-mTor signaling was modified in T17M RHO CASP-7 retina as well leading to $35 \%$ upregulation of Akt1 and $49 \%$ downregulation of mTor mRNAs. Similar to in vitro experiment suggesting the modulation of the TRAF2-JNK signaling, in vivo we observed 


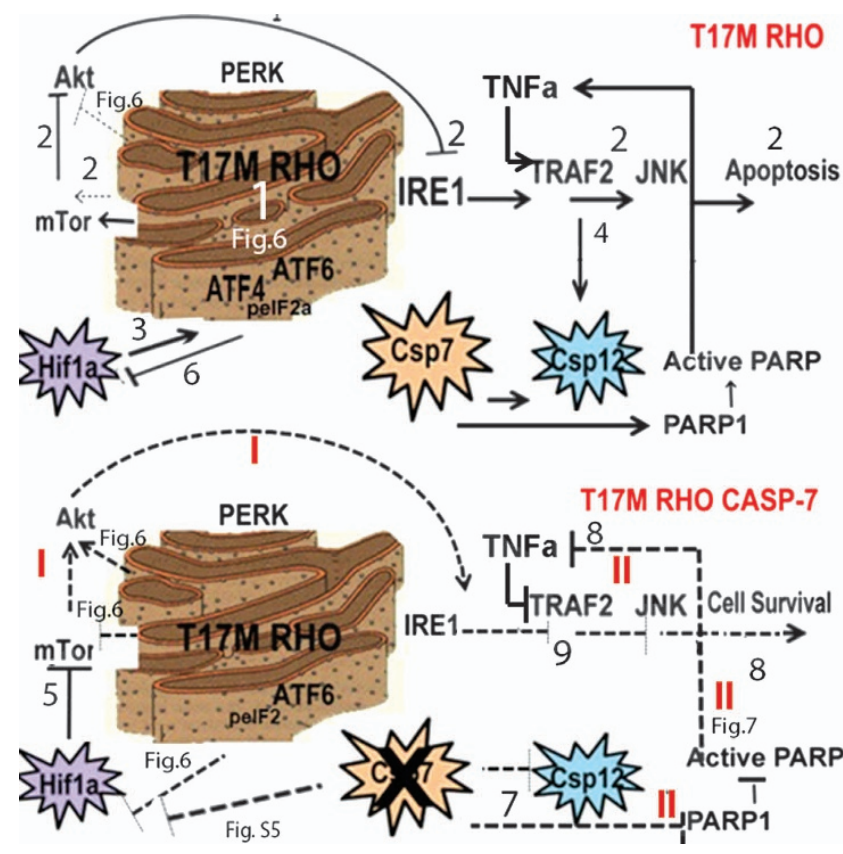

Figure 8 The proposed therapeutic mechanism of caspase-7 ablation in ADRP T17M RHO retinas. Upper: the expression of mutant T17M RHO protein in ADRP photoreceptors leads to the activation of the ER stress response (1, Kunte et al. ${ }^{7}$ ), upregulation of $\mathrm{mTOR}$, inhibition of pAkt and consequently upregulation of IRE1-JNK apoptosis (2, Kato et al. ${ }^{28}$ and Figures 5 and 6 ). In addition, the Hif1a protein (Figure 6) is upregulated, perhaps resulting in activation of the PERK pathway through translational regulation of ATF4 (3, Blais et al. $\left.{ }^{20}\right)$, and TNFa is increased in T17M RHO retinas (Figure 6). The activated ER stress response leads to recruitment of TRAF2 and consequently, upregulation of the cleaved caspase-12 protein (4, Yoneda et al. ${ }^{26}$ and Figures 5 and 6 ), which is known to precede caspase7 cleavage during apoptosis. Bottom: caspase- 7 ablation in photoreceptors expressing the T17M RHO protein leads to a reprogramming of the ER stress response and downregulation of apoptosis. Two ways for regulation of apoptosis are proposed. I: The first is associated with reprogramming UPR-induced gene and protein expression. Caspase-7 ablation mitigates ER stress response modulating the ER stress-induced gene and protein expression (Figures 5and 6). This results in downregulation of mTOR and consequently, activated Akt protein (Figures 5 and 6 ). Activated AKT in turn inhibits the TRAF2-JNK apoptosis (Figure 7). Independently, the downregulation of mTor could occur through inhibition of HIF1 (5, Wilson and $\mathrm{Hay}^{30}$ ) that in turn could be downregulated either by caspase-7 ablation (Figure 6 and Supplementary Figure S5) or by PERK signaling (6. Papadakis et al. $\left.{ }^{31}\right)$. II: Diminishing of TRAF2- JNK apoptosis could also result from the downregulation of TNFa and PARP1. Ablation of caspase-7 deactivates the PARP protein (7, Boucher et $a l . .^{32}$ and Figure 7$)$ that has been shown to reduce TNFa (8, Garcia et al. ${ }^{33}$ and Figure 7). TNFa has been demonstrated to reduce the JNK-dependent apoptosis through TNFTNFR1- TRADD-TRAF2-RIP-TAK1-IKK signaling (9, Jackson-Bernitsas et al. ${ }^{27}$ and Figure 7)

$27 \%$ reduction of Traf2 mRNA in T17M RHO CASP-7 photoreceptors. The apoptotic caspase- 12 and caspase-3 mRNAs were downregulated by 44 and $32 \%$, respectively.

Protein analysis demonstrated that ER stress-associated genes, such as PATF6 and Atf 4 were decreased by $57 \%$ and $55 \%$, respectively. The expression of the pro-survival gene pAkt was increased in $\mathrm{P} 30 \mathrm{~T} 17 \mathrm{M}$ RHO CASP-7 retinas by $60 \%$. In contrast, the mTOR protein expression was downregulated by $38 \%$. Additionally, the T17M RHO retina demonstrated an increase in TRAF2 by $217 \%$, which was diminished by $31 \%$ in T17M RHO CASP-7 retina.
Caspase-7 ablation in T17M RHO retina leads to a decrease in hif1a protein production. Analysis of the T17M RHO CASP-7 retinal protein extract also revealed that the Hif1a protein was dramatically reduced by $77 \%$ compared with T17M RHO and by $84 \%$ compared with wt (Figure 6; Supplementary Table S1). Therefore, we wanted to determine if the modulation in the $\mathrm{Hf1a}$ protein was specific to caspase- 7 ablation.

Previously we have reported that during the progression of ADRP, Hif1 gene expression is upregulated in transgenic retinas $^{5,6}$ and that this elevation may be associated with the activated UPR. Therefore, we decided to test if during the reprogramming of UPR-induced gene expression in vivo, modulation of the Hif1a protein and knockdown of caspase-7 expression are linked. In the literature, it has been demonstrated that expression of the ATF4 protein (PERK pathway) could be modulated by hypoxia. ${ }^{20}$ To verify this hypothesis, we conducted an experiment with cells co-transfected with human HIf1 cDNA and cont. or Csp7-siRNAs (Supplementary Figure S5). Our results demonstrated a reduction of $\mathrm{Hf} 1 \mathrm{a}$ protein by $59 \%$ in Hif1a + Csp7-siRNA cells. In addition, this decrease was associated with a $66 \%$ decline in the level of ATF4 protein.

Caspase-7 ablation in T17M RHO retina reprograms photoreceptor cell death via downregulation of PARP1TNFa-TRAF2-c-JUN. We decided to determine the level of apoptotic signaling upstream of the ER-associated caspase-7. The T17M RHO retina demonstrated an increase in the pcJUN protein by $236 \%$ that was significantly diminished by $50 \%$ in T17M RHO CASP-7 retina (Figure 6b; Supplementary Figure S1 and Supplementary Table S1). Taking a closer look at the mechanism of cell death in T17M RHO retina, we determined that protein levels of the inflammatory pro-death marker TNFa were dramatically increased by $235 \%$ in $\mathrm{T} 17 \mathrm{M}$ $\mathrm{RHO}$ retina compared with wt (Figure 7). Caspase-7 ablation, however, resulted in reduction of TNFa by $72 \%$ compared with T17M RHO retina. Another pro-apoptotic marker, activated PARP1 was elevated by 1.8 -fold in ADRP retinas. Again, caspase-7 ablation led to a $52 \%$ reduction of activated PARP1 in $\mathrm{T} 17 \mathrm{M} \mathrm{RHO}$ retina.

\section{Discussion}

The ER stress-associated caspase-7 has been implicated with retinal degeneration in animal models of ADRP. ${ }^{5,6} \mathrm{We}$ therefore sought to determine whether caspase-7 ablation could be therapeutic in T17M RHO retinas. Here, we hypothesized that the deficit in caspase-7 would delay deterioration of retinal structure/function and slow down progressive degeneration, thus protecting retinas from lightinduced damage through activation of pro-survival pathways, that would lead to a reduction in ER stress and apoptosis. We validated all these points and demonstrated that caspase-7 ablation in T17M RHO retina delayed retinal degeneration via modulation of the ER stress response leading to decreased apoptosis.

Although caspase- 7 and caspase- 3 are both downstream executioner proteases, the elimination of caspase- 3 has been shown to provide only minimal and transient photoreceptor 
protection in $r d-1 .^{21}$ While the cleavage of caspase-7 is upregulated during ADRP (Figure 1), the role of caspase-7 and UPR activation in retinal degeneration have not been previously explored. Therefore, we examined the effect of caspase-7 ablation in T17M RHO mice on retinal structure and function. We found that ONL thickness was rescued and that a-wave amplitudes of the scotopic ERG were protected in these retinas (Figure $2 \mathrm{a}$ ). While the $\mathrm{b}$-wave amplitudes were increased in P30-P90 only from $145 \%$ to $182 \%$, the a-wave amplitudes were elevated more markedly. Apparently, this phenomenon is associated with the fact that ADRP photoreceptors are the first to degenerate and the first to respond favorably to therapy. It is also important to note that while this significant improvement still does not reach the level found in wt, the functional preservation in T17M RHO CASP-7 photoreceptors was marked even at 3 months (age).

In addition to functional improvements, we observed a preservation of retinal structure. The T17M RHO mice are characterized by a slightly more rapid retinal degeneration in the inferior hemisphere (Figure $3 b$ ) than in the superior retina. The lack of caspase-7 in P30 T17M RHO mice slowed down the deterioration of the photoreceptors and significantly preserved the integrity of the neuronal retina. The inferior region of T17M RHO CASP-7 retinas responded more dramatically to the therapy, and this suggests a different extent of cellular signaling responsible for the deterioration of the photoreceptors in these two regions.

The histological analysis revealed proportional loss of photoreceptors from $\mathrm{P} 30$ to $\mathrm{P} 90$ in $\mathrm{T} 17 \mathrm{M} R \mathrm{RHO}$ retina that was in agreement with the ERG and OCT data. Interestingly, the $\mathrm{P} 30$ and $\mathrm{P} 90 \mathrm{~T} 17 \mathrm{M}$ RHO CASP-7 retinas did not demonstrate this trend and had the same number of nuclei over 3 months. This fact indicates the importance of the histological analysis in evaluation of retinal structure and suggests other potential changes that could occur in the retina and be detected by SD-OCT.

The protective role of caspase-7 ablation in T17M RHO retinas is obvious when analyzing the functional preservation of light-treated ADRP photoreceptors. For example, the a-wave ratio in the T17M $R H O$ mice was diminished by $33 \%$. These data are in agreement with the study of White et al., ${ }^{4}$ who demonstrated the sensitivity of T17M RHO ERG responses and the apoptotic signal to light exposure (Figure 4). The ablation of caspase-7, however, protects these mice from the cellular stress leading to significantly reduced levels of apoptosis that are similar to wt. Thus, this experiment also suggests that the activation of caspase-7 significantly contributes to light-induced DNA fragmentation and apoptosis, which have been described to occur via ER stress activation ${ }^{22}$ and C-JUN-induced apoptosis. ${ }^{23}$

We were very intrigued by the fact that genetic manipulation of $\mathrm{T} 17 \mathrm{M} \mathrm{RHO}$ leads to a reprogramming of apoptosis and decided to test the pro-inflammatory properties of dying cells. We found that the level of TNF $\alpha$ is upregulated in T17M RHO retina and that caspase- 7 ablation leads to a reduction in $\mathrm{TNF} \alpha$. This fact suggests that both necrotic and apoptotic upregulation might occur in T17M RHO retinas because TNFa is known to be a marker for both cell death pathways. To answer the question of whether necrosis is involved in ADRP progression, $\mathrm{T} 17 \mathrm{M}$ RHO retinas will have to be examined for RIP3 ${ }^{24}$ expression as had previously been done for rd10 mice. ${ }^{25}$

How does caspase-7 ablation provide the therapeutic effect? To answer this question, we performed in vivo and in vitro studies, and found very similar results demonstrating that the UPR-induced gene expression is modified. In T17M $\mathrm{RHO}+$ Csp7-siRNA cells, the Atf4, Atf6, Bip, Chop, Cnx and Hsp90 are significantly reduced (Figure 5). The level of ER stress-associated caspase-12 gene expression and its activity are also significantly diminished. This fact could affect the Traf2 gene and protein expression that is known to be a binding partner of pro-Csp12. ${ }^{26}$ In addition, Traf2 could be diminished by reduced TNFa-TNFR1-TRADD-TRAF2-c-JUN signaling as has been proposed. ${ }^{27}$ Similar modulation of the UPR observed in the tunicamycin-treated cells deficient in caspase-7 suggests that the caspase-7 has a much more general role than reprogramming the cellular signaling in T17M RHO photoreceptors and much broader potential applications in UPR regulation. However additional experiments will have to be conducted to answer the question of how exactly caspase-7 ablation reprograms the UPR-induced protein network.

With regards to mTor, we learned that the mTor gene and protein expression are diminished in both cells treated with T17M RHO + Csp7-siRNA cells and T17M RHO CASP-7 retina (Figures 5 and 6). Moreover, in T17M RHO CASP-7 mice, we observed the elevation of pAkt (Figure 6), suggesting negative regulation by $\mathrm{mTor}$. The role of a negative feedback loop initiated by mTORC1 in AKT activation leading to induction of ER stress-associated apoptosis via selective activation of the IRE-JUN pathway has been recently proposed. $^{28}$

In T17M RHO CASP-7 retinas, we observed a downregulation of the Hif1a protein (Supplementary Figure S5). Although the possible role of caspase-7 in the regulation of hypoxia-induced apoptosis was recently investigated, ${ }^{17}$ we demonstrated a reverse link between these two molecules. Our in vitro experiments suggested that the ablation of caspase-7 leads to a reduction of Hif1a. The HIF1a could cause a rapid activation of the UPR through negative regulation of its $\mathrm{mTor}$ target ${ }^{29}$ and $\mathrm{ATF} 4,{ }^{31}$ thus perhaps leading to a modified ER stress response. Therefore, these data also imply that during hypoxia, which results in the upregulation of caspase-7 and DNA fragmentation, downregulating caspase-7 could also modulate apoptosis via Hif1a and the PERK-ATF4-CHOP signaling pathway.

Finally, we found that the ablation of caspase-7 leads to reduction of activated pro-apoptotic PARP1 (Figure 7), the proteolysis of which is known to be promoted by $\mathrm{N}$-terminal exosite of caspase-7. ${ }^{32}$ Therefore, in the absence of caspase-7, a reduction in pro-apoptotic PARP1 could significantly contribute to the reprograming of apoptosis. Additionally, the inhibition of PARP1 has been shown to reduce TNFa and modulate apoptosis. ${ }^{33}$ Together our data support this hypothesis allowing us to propose PARP1-TNF $\alpha$-TRAF2-JNK signaling as the mode for downregulation of apoptosis.

Here, we explored the possible protein regulatory network involved in the rescue of T17M RHO photoreceptors and proposed that caspase-7 ablation modulates cell signaling in degenerating retinas (Figure 8), thus promoting photoreceptor 
cell survival. However, the degree of cell survival demonstrated did not reach wt levels, suggesting that other cellular pathways are involved in the mechanism of ADRP pathogenesis. The first possible survival pathway is associated with the downregulation of Hif1a, the reprogramming UPR and the inhibition of mTor targets, thus blocking apoptosis via the activation of AKT and inhibition of Traf2-C-JUN signaling. The second pathway is proposed to negatively regulate apoptosis through inhibition of PARP1 leading to diminished TNF $\alpha$-TRAF2-pc-JUN signaling. These two signaling pathways could act synergistically or be activated individually. In both scenarios, a reduction in c-Jun apoptosis would lead to ADRP photoreceptor survival.

\section{Materials and Methods}

Mice. Transgenic mice expressing human T17M RHO, wt mice and caspase$7-1$ - (CASP-7) mice were used for this study. The animal protocol was approved by the University of North Texas Health Center Animal Care and Use Committee and was conducted following the animal guidelines according to the ARVO statement for the Use of Animals in Ophthalmic and Vision Research. All mice were raised under a 12-h light/12-h dark cycle. To obtain T17M RHO CASP-7 mice, we crossed T17M RHO mice with CASP-7 mice purchased from Jackson, Bar Harbor, ME, USA (http://www.jax.org). Caspase-7 - / - (CASP-7) mice have a normal appearance, organ morphology and lymphoid and eye development. The genotype was determined using PCR analysis with human RHO-specific primers (sense: $5^{\prime}$-GAGTGCACCCTCCTTAGGCA-3' and antisense: $5^{\prime}$-TCCTGACTCGAGGACCCTAC-3') that amplified a single band of $\sim 300$ bp in the human Rho transgene. To identify the Casp-7 - / - genotype, PCR was performed using four primers (primer 1: $5^{\prime}$-TGCTAAAGCGCATGCTCC AGACTG-3', primer 2: 5'-ATCCTTTATGGGTGTCACGCC-3'; primer 3: 5'-GA CTGCTTCCACAGCCTCTAACTG-3' and primer 4: $5^{\prime}$-GTCTGGTAAAGTGGCGG AGGACG- $3^{\prime}$ ) that amplified a 240-bp fragment indicating the presence of the fusion transgene. Primers 3 and 4 provided an internal control and amplified a 344-bp fragment. The presence of two bands indicated a positive result.

Transfection of $661 \mathrm{~W}$ cells. For the in vitro experiments, we used a pCMV6-AC-GFP (Rockville, MD, USA) plasmid expressing the hT17M RHO cDNA fused to the GFP protein on its C-terminal. The Mirus kit with fluorescence dye Cy3 was used to label the control and Csp7-siRNA (MIR 8750, Mirus Bio, Madison, WI, USA). The cone-derived cell line $661 \mathrm{~W}$ was used. These cells were maintained in Dulbecco's modified Eagle's medium (low glucose) supplemented with $10 \% \mathrm{FBS}$ and $1 \%$ penicillin-streptomycin. The cultures were incubated at $37^{\circ} \mathrm{C}$ in $5 \% \mathrm{CO}_{2}$. The $\mathrm{Csp} 7$-siRNA and control siRNA were purchased from Dharmacon, Waltham, MA, USA. Both siRNAs were labeled with Cy3 (Rhodamine) using a Mirus kit. The cells were co-transfected with $20 \mathrm{nM}$ of siRNA and $10 \mu \mathrm{g}$ of pCMV6-AC-T17M RHO-GFP using Lipofectamine 2000 (Invitrogen, Grand Island, NY, USA) according to the manufacturer's protocol. After $48 \mathrm{~h}$ of transfection, the cells were harvested. The GFP- and Cy3-positive cells were collected using FACS. The protein extracts were prepared (Supplementary Information).

Plasmid and construct. Human RHO cDNA was cloned into the pCMV6-ACGFP vector at the Notl sites (OriGene, Rockville, MD, USA). The GFP protein was inserted before the stop codon to produce a fused hRHO-GFP. protein. The T17M RHO was generated using the QuikChange Site-Directed Mutagenesis Kit (Stratagene, La Jolla, CA, USA). The plasmid is referred to as pCMV6-AC-hT17M RHO-GFP

RNA and protein extraction. Whole retinas were collected from individual wt, T17M RHO, T17M RHO CASP-7 mice at postnatal days (P) 12, 15, 18, 21, 25 and 30. The RNA was extracted using RNeasy mini prep kits (Qiagen, Valencia, CA, USA). After treating the RNA with DNasel (Invitrogen, Grand Island, NY USA), the RNA was converted to CDNA using Super Script II Reverse Transcriptase (Invitrogen).

The total protein was isolated from wild-type and transgenic retinas. Retinal protein extracts were obtained from dissected retinas by sonication in a buffer containing $25 \mathrm{~mm}$ sucrose, $100 \mathrm{~mm}$ Tris- $\mathrm{HCl}, \mathrm{pH} 7.8$, and a mixture of protease and phosphatase inhibitors (Halt Protease and Phosphatase Inhibitor Cocktail, Thermo
Scientific, Rockford, IL, USA). The protein concentration was measured using a BioRad protein assay, and an equal concentration of total protein was loaded onto 12 or $15 \%$ SDS-PAGE. The proteins were transferred to polyvinylidene difluoride membranes (Invitrogen) by electrophoresis. Then, the primary antibodies were applied. The secondary antibodies were tagged with infrared dyes. The detection of proteins was performed using an infrared imager (Li-Cor, Inc., Lincoln, NE, USA).

qRT-PCR. We used a custom TaqMan array plate with 32 genes, including Gapdh as the endogenous control (Applied Biosystems, Carlsbad, CA, USA). RTPCR with the TaqMan universal PCR master mix (Applied Biosystems) and the StepOnePlus Real-Time PCR system (Applied Biosystems) was performed as described. ${ }^{7}$ Fold differences were calculated using $R Q$.

Antibodies. Anti-phosphorylated c-Jun (1:1000); anti-mTor (1:1000); anticleaved caspase-7 and anti-caspase-7 (1:1000); anti-TRAF2 (1:1000); PARP1 (1:1000) (from Cell Signaling Technology, Inc., Danvers, MA, USA); antiphosphorylated cleaved Atf6 $(1: 1000)$ (Imgenex, San Diego, CA, USA); anticaspase-12 (1: 1000); anti-Chop (1:1000); anti-ATF6 (1: 1000), anti-pAKT (1: 1000) (Abcam, Co., Cambridge, MA, USA); anti-TNFa $(1 ; 1000)$ and anti-Bip $(1: 1000)$ (Santa-Cruz Biotechnology, Santa Cruz, CA, USA) and anti-Hif1a (1:500) (Bethyl Laboratories Inc., Montgomery, TX, USA), anti- $\beta$-actin 1:1000 (Sigma-Aldrich, St Louis, MO, USA). Anti-rhodopsin (1D4) (University of British Columbia, Vancouver, $\mathrm{BC}$, Canada) primary antibody and peanut agglutinin Biotin-conjugated (PNA) (Vector Labs, Burlingame, CA, USA) were used in immunohistochemistry.

Light-induced experiment and ELISA quantification of apoptosis. The light-induced damage of the retina was performed using bright white light and the method described previously. ${ }^{4,34}$ After exposure, ERG was performed on mice dark adapted for $12 \mathrm{~h}$ to test the photoreceptor response. A nucleosome release assay was used to measure levels of apoptosis in retinal specimens using the Cell Death Detection ELISA (Roche Diagnostics, Indianapolis, IN, USA). We quantified the DNA fragmentation resulting from apoptosis in transgenic, knockout and wt retinas. The $\mathrm{Ca}^{2+}$ - and $\mathrm{Mg}^{2+}$-dependent nuclease cleavage of the double-stranded DNA resulted in the release of mono- and oligonucleosomes, and these complexes are tightly associated with the core histones $\mathrm{H} 2 \mathrm{~A}, \mathrm{H} 2 \mathrm{~B}, \mathrm{H} 3$ and $\mathrm{H} 4$. Therefore, we quantified nucleosome release levels using a sandwich-enzyme immunoassay with mouse monoclonal antibodies directed against DNA and histones and a Cell Death Detection ELISA kit. Individual right and left retinas were harvested and processed according the manufacturer's procedure. The retinas were placed in $200 \mu$ l of lysis buffer (provided with the kit) on ice and were homogenized for $3 \mathrm{~s}$ with a tissue homogenizer (Polytron; PT 1200, Radnor, PA, USA). The homogenates were centrifuged at $200 \times g$ for $10 \mathrm{~min}$, and $10 \mu \mathrm{l}$ of the resulting supernatant were used for further dilution into $990 \mu \mathrm{l}$ of lysis buffer. A volume of $20 \mu \mathrm{l}$ of this final dilution was used in the assay.

Electroretinography. We performed the scotopic ERG analysis with darkadapted (12 h) P30, P60 and P90 mice using LKC Technologies, Gaithersburg, MD, USA, as previously described. ${ }^{7}$ The mice were anesthetized with an intraperitoneal injection of $50 \mathrm{mg}$ xylazine/ $\mathrm{kg}$ body weight and $50 \mathrm{mg}$ ketamine $/ \mathrm{kg}$ body weight. The mouse corneas were anesthetized locally with $0.5 \%$ proparacaine hydrochloride (Bausch \& Lomb, Rochester, NY, USA), and the pupils were dilated with $2.5 \%$ phenylephrine hydrochloride (Bausch \& Lomb). The ground and reference electrodes were inserted subdermally by the hind limb and centered along the nasal ridge, respectively. Gold loop electrodes were placed on each eye with a drop of $2.5 \%$ hypromellose. The scotopic ERGs were registered with $10 \mu$ s flashes of white light at $-20,-10,0,5,10$ and $15 \mathrm{~dB}$.

Spectra domain-optical coherent tomography. The SD-OCT in the P30, P60 and P90 animals was performed using the Spectral Domain Ophthalmic Imaging System (SDOIS) (Bioptigen, Morrisville, NC, USA). The mice were anesthetized. Horizontal volume scans through the area dorso-temporal from the optic nerve (superior retina) and the area ventro-temporal from the optic nerve (inferior retina) were used to evaluate the thickness of the ONL. For measuring the thickness of the ONL, six calibrated calipers were placed in the superior and inferior hemispheres of retinas within $100,200,300$ and $400 \mu \mathrm{m}$ of the optic nerve head. The thickness of the ONL was determined by averaging ten measurements.

Histology. For hematoxylin and eosin staining, mouse eyes were enucleated at 1 and 3 months of age and were fixed overnight in $4 \%$ of freshly made 
paraformaldehyde in phosphate-buffered saline (PBS). Afterwards, eye cups were transferred to PBS to remove formaldehyde and submerged sequentially in solutions of $10 \%, 20 \%$ and $30 \%$ sucrose for at least $1 \mathrm{~h}$ each. Eye cups were then embedded in cryostat compound (Tissue TEK OCT, Sakura Finetek USA, Inc., Torrance, CA, USA) and frozen at $-80^{\circ} \mathrm{C}$. Also, 12-micron sections were obtained by using cryostat. Slides with right and left retinas were used for further histological analysis. To count the nuclei of photoreceptors, we stained cryostat sectioned retinas with hemotaxylin and eosin. Digital images of right and left retinas of individual mice were analyzed in the central superior and inferior equally located from the optic nerve head. Images were analyzed by a masked investigator.

Statistical analysis. To perform statistical analysis in the light-exposure experiment, we calculated the b- to a-wave ratio amplitudes from the scotopic $E R G$ in the $R$ and $L$ eyes individually, and the $R / L$ ratio of the $B / A$ waves was taken for comparison. A one-way ANOVA was used to calculate the difference in the apoptotic signal between the right (light exposed) and left (control) eyes (R/L apoptotic signal). A one-way ANOVA was also used in the c-Jun and TNF western blot protein analysis. A two-way ANOVA was used to perform statistical analysis in the caspase-7 gene expression assay, the ERG and the OCT. A $t$-test was used to calculate significant differences in the western blot analysis and gene expression assay of the 661W cells transfected with hT17M RHO-GFP and Csp7siRNA and the T17M RHO and T17M RHO CASP-7 retinas. For all experiments, a $P$-value higher than 0.05 was considered significant $\left({ }^{*} P<0.05\right.$, ${ }^{\star *} P<0.01$, $\left.{ }^{* * *} P<0.001,{ }^{* * *} P<0.0001\right)$.

\section{Conflict of Interest}

The authors declare no conflict of interest.

Acknowledgements. We thank Dr. Kunte for technical support and Dr. Al-Ubaidi for the 661W cells. This work was supported by the NIH (R01EY020905), the Foundation Fighting Blindness (TA-GT-0409-0508-NTERI), Hope for Vision and DOD W81XH-10-2-0003.

1. Mendes HF, van der Spuy J, Chapple JP, Cheetham ME. Mechanisms of cell death in rhodopsin retinitis pigmentosa: implications for therapy. Trends Mol Med 2005; 11 : 177-185.

2. Krebs MP, Holden DC, Joshi P, Clark CL 3rd, Lee AH, Kaushal S. Molecular mechanisms of rhodopsin retinitis pigmentosa and the efficacy of pharmacological rescue. $\mathrm{J} \mathrm{Mol} \mathrm{Biol}$ 2010; 395: 1063-1078.

3. Chang GQ, Hao Y, Wong F. Apoptosis: final common pathway of photoreceptor death in rd, rds, and rhodopsin mutant mice. Neuron 1993; 11: 595-605.

4. White DA, Fritz JJ, Hauswirth WW, Kaushal S, Lewin AS. Increased sensitivity to light-induced damage in a mouse model of autosomal dominant retinal disease. Invest Ophthalmol Vis Sci 2007; 48: 1942-1951.

5. Shinde VM, Sizova OS, Lin JH, Lavail MM, Gorbatyuk MS. ER stress in retinal degeneration in S334ter Rho rats. PLoS One 2012; 7: e33266.

6. Gorbatyuk MS, Knox T, LaVail MM, Gorbatyuk OS, Noorwez SM, Hauswirth WW et al. Restoration of visual function in $\mathrm{P} 23 \mathrm{H}$ rhodopsin transgenic rats by gene delivery of BiP/Grp78. Proc Natl Acad Sci USA 2010; 107: 5961-5966.

7. Kunte MM, Choudhury S, Manheim JF, Shinde VM, Miura M, Chiodo VA et al. ER stress is involved in T17M rhodopsin-induced retinal degeneration. Invest Ophthalmol Vis Sci 2012; 53: 3792-3800.

8. Henshall DC, Skradski SL, Meller R, Araki T, Minami M, Schindler CK et al. Expression and differential processing of caspases 6 and 7 in relation to specific epileptiform EEG patterns following limbic seizures. Neurobiol Dis 2002; 10: 71-87.

9. Juan TSC, McNiece IK, Argento JM, Jenkins NA, Gilbert DJ, Copeland NG et al. Identification and mapping of Casp7, a cysteine protease resembling CPP32 beta, interleukin-1 beta converting enzyme, and CED-3. Genomics 1997; 40: 86-93.

10. Zhang $Y$, Goodyer $C$, LeBlanc $A$. Selective and protracted apoptosis in human primary neurons microinjected with active caspase-3,-6,-7, and-8. J Neurosci 2000; 20: 8384-8389.

11. Walsh JG, Cullen SP, Sheridan C, Luthi AU, Gerner C, Martin SJ. Executioner caspase-3 and caspase-7 are functionally distinct proteases. Proc Natl Acad Sci USA 2008; 105 . 12815-12819.

12. Larner SF, Hayes RL, McKinsey DM, Pike BR, Wang KK. Increased expression and processing of caspase-12 after traumatic brain injury in rats. J Neurochem 2004; 88: 78-90.
13. Lakhani SA, Masud A, Kuida K, Porter GA Jr, Booth CJ, Mehal WZ et al. Caspases 3 and 7: key mediators of mitochondrial events of apoptosis. Science 2006; 311: 847-851.

14. Le DA, Wu Y, Huang Z, Matsushita K, Plesnila N, Augustinack JC et al. Caspase activation and neuroprotection in caspase-3- deficient mice after in vivo cerebral ischemia and in vitro oxygen glucose deprivation. Proc Natl Acad Sci USA 2002; 99: 15188-15193.

15. Nicolini G, Rigolio R, Miloso M, Bertelli AA, Tredici G. Anti-apoptotic effect of transresveratrol on paclitaxel-induced apoptosis in the human neuroblastoma SH-SY5Y cell line. Neurosci Lett 2001; 302: 41-44.

16. Kaur J, Mencl S, Sahaboglu A, Farinelli P, van Veen T, Zrenner E et al. Calpain and PARP activation during photoreceptor cell death in $\mathrm{P} 23 \mathrm{H}$ and $\mathrm{S} 334$ ter rhodopsin mutant rats. PLoS One 2011; 6: e22181.

17. Eguchi R, Tone S, Suzuki A, Fujimori Y, Nakano T, Kaji K et al. Possible involvement of caspase- 6 and -7 but not caspase- 3 in the regulation of hypoxia-induced apoptosis in tubeforming endothelial cells. Exp Cell Res 2009; 315: 327-335.

18. Mao H, Gorbatyuk MS, Rossmiller B, Hauswirth WW, Lewin AS. Long term rescue of retinal structure and function in $\mathrm{P} 23 \mathrm{H} \mathrm{RHO}$ transgenic mice by rhodopsin RNA replacement with a single AAV vector. Hum Gene Ther 2012; 23: 356-366.

19. Millington-Ward S, O'Neill B, Tuohy G, Al-Jandal N, Kiang AS, Kenna PF et al. Strategems in vitro for gene therapies directed to dominant mutations. Hum Mol Genet 1997; 6: $1415-1426$.

20. Blais JD, Filipenko V, Bi M, Harding HP, Ron D, Koumenis C et al. Activating transcription factor 4 is translationally regulated by hypoxic stress. Mol Cell Biol 2004; 24 : 7469-7482.

21. Zeiss CJ, Neal J, Johnson EA. Caspase-3 in postnatal retinal development and degeneration. Invest Ophthalmol Vis Sci 2004; 45: 964-970.

22. Yang LP, Wu LM, Guo XJ, Li Y, Tso MO. Endoplasmic reticulum stress is activated in lightinduced retinal degeneration. J Neurosci Res 2008; 86: 910-919.

23. Roduit R, Schorderet DF. MAP kinase pathways in UV-induced apoptosis of retinal pigment epithelium ARPE19 cells. Apoptosis 2008; 13: 343-353.

24. Zhang J, Dong Z, Yu J, Chi N, Tao L, Li X et al. Simplified coherent receiver with heterodyne detection of eight-channel $50 \mathrm{~Gb} / \mathrm{s}$ PDM-QPSK WDM signal after $1040 \mathrm{~km}$ SMF-28 transmission. Opt Lett 2012; 37: 4050-4052.

25. Murakami $Y$, Matsumoto $H$, Roh M, Suzuki J, Hisatomi T, Ikeda $Y$ et al. Receptor interacting protein kinase mediates necrotic cone but not rod cell death in a mouse model of inherited degeneration. Proc Natl Acad Sci USA 2012; 109: 14598-14603.

26. Yoneda T, Imaizumi K, Oono K, Yui D, Gomi F, Katayama T et al. Activation of caspase-12, an endoplastic reticulum (ER) resident caspase, through tumor necrosis factor receptorassociated factor 2-dependent mechanism in response to the ER stress. J Biol Chem 2001; 276: $13935-13940$.

27. Jackson-Bernitsas DG, Ichikawa H, Takada $Y$, Myers JN, Lin XL, Darnay BG et al. Evidence that TNF-TNFR1-TRADD-TRAF2-RIP-TAK1-IKK pathway mediates constitutive NF-kappaB activation and proliferation in human head and neck squamous cell carcinoma. Oncogene 2007; 26: 1385-1397.

28. Kato H, Nakajima S, Saito Y, Takahashi S, Katoh R, Kitamura M. mTORC1 serves ER stress-triggered apoptosis via selective activation of the IRE1-JNK pathway. Cell Death Differ 2012; 19: 310-320.

29. Wouters BG, Koritzinsky M. Hypoxia signalling through mTOR and the unfolded protein response in cancer. Nat Rev Cancer 2008; 8: 851-864.

30. Wilson WR, Hay MP. Targeting hypoxia in cancer therapy. Nat Rev Cancer 2011; 11 393-410.

31. Papadakis Al, Paraskeva E, Peidis P, Muaddi H, Li S, Raptis L et al. elF2\{alpha\} Kinase PKR modulates the hypoxic response by Stat3-dependent transcriptional suppression of HIF-1\{alpha\}. Cancer Res 2010; 70: 7820-7829.

32. Boucher D, Blais V, Denault JB. Caspase-7 uses an exosite to promote poly(ADP ribose) polymerase 1 proteolysis. Proc Natl Acad Sci USA 2012; 109 : 5669-5674.

33. Garcia S, Bodano A, Pablos JL, Gomez-Reino JJ, Conde C. Poly(ADP-ribose) polymerase inhibition reduces tumor necrosis factor-induced inflammatory response in rheumatoid synovial fibroblasts. Ann Rheum Dis 2008; 67: 631-637.

34. Krebs MP, White DA, Kaushal S. Biphasic photoreceptor degeneration induced by light in a T17M rhodopsin mouse model of cone bystander damage. Invest Ophthalmol Vis Sci2009; 50: $2956-2965$

(i) () $\Theta$ Cell Death and Disease is an open-access journal published by Nature Publishing Group. This work is licensed under the Creative Commons Attribution-NonCommercial-No Derivative Works 3.0 Unported License. To view a copy of this license, visit http://creativecommons.org/licenses/by-nc-nd/3.0/ 